\title{
InAs/GaAs Quantum Dot Lasers Monolithically Integrated on Group IV Platform
}

\author{
Keshuang $\mathrm{Li}^{1}$, Mingchu Tang ${ }^{1}$, Mengya Liao ${ }^{1}$, Jiang $\mathrm{Wu}^{1}$, Siming Chen ${ }^{1}$, Alwyn Seeds ${ }^{1}$, and Huiyun Liu ${ }^{1}$ \\ ${ }^{1}$ Department of Electronic and Electrical Engineering, University College London, London, email: Huiyun.liu@ ucl.ac.uk
}

\begin{abstract}
III-V quantum dot lasers monolithically integrated on silicon platform attracts intensive interests due to its advantages on providing a promising solution for reliable and efficient light source to integrated on photonics and electronics circuits. Compared to wafer bonding technique, monolithic integration its more attractive for large scale, low cost and streamline fabrication. In this paper, we give a brief review on our recent progress of III-V quantum dot lasers monolithically integrated on $4^{\circ}$ offcut and exact (001) Si substrates for the silicon photonic integration.
\end{abstract}

\section{INTRODUCTION}

Monolithic integration of III-V reliable and efficient electrically pumped continues wave (CW) semiconductor laser on silicon platform is an ultimate solution for producing complex Si based optoelectronics integrated circuits. Directly epitaxial growth for III-V semiconductor material on silicon substrate gives a promising solution to provide a low cost, large scale and high yield on-chip light source. However, large material dissimilarities between group IV and group III-V materials cause many significant challenges for monolithic integration. For example, large material mismatch, thermal expansion ecoefficiency difference and polar on non-polar growth which cause various types of defects including threading dislocations (TDs), microcracks and antiphase boundaries (APBs). These may generate non-radiative recombination centres which will dramatically decrease the performance of fabricated devices. To overcome these challenges, many approaches including dislocation filter layers (DFLs) and thermal annealing (TA) have been considered in our results in order to improve device performance. Considering the active region, quantum dot structure attracts extensive research interests in past several decades for the monolithic integration of III-V on Si. It has been widely used as active layers in semiconductor lasers due to the advantages of low threshold current density $\left(\mathrm{J}_{\mathrm{th}}\right)$, insensitive to TDs, and high temperature stability. In this paper, we will give a review of our recent progress on III-V quantum dot lasers monolithically integrated on Si platform.

\section{QUNATUM DOT LASERS MONOLITHICALLY INTEGRATED ON OFFUCT SILICON SUBSTRATE}

In this work, InAs/GaAs quantum dot lasers structure was directly grown on $4^{\circ}$ offcut to [011] Si substrate using solidsource molecular beam epitaxy (MBE) system to prevent the formation of APBs. In our previous work, we have reported the first electrically pumped InAs/GaAs quantum dot laser directly grown on Si substrate by using a optimized GaAs nucleation layer on $\mathrm{Si}$ and a $\mathrm{InGaAs} / \mathrm{GaAs}$ strained layer superlattice (SLS) as DFLs [1]. After that, we consistently improve the devices performance in our following works. Eventually, due to the high-quality GaAs film we realized on offcut Si substrate by applying combined strategies as AlAs nucleation layer, optimized InGaAs/GaAs DFLs and in-situ thermal annealing. A high performance $1310 \mathrm{~nm}$ InAs/GaAs quantum dot laser monolithically grown on offcut $\mathrm{Si}$ substrate with record low threshold current density $62.5 \mathrm{~A} / \mathrm{cm}^{2}$ at room temperature (RT) has been demonstrated [2]. The maximum output power of single facet is about $105 \mathrm{~mW}$ and the maximum operation temperature is up to $75^{\circ} \mathrm{C}$ under $\mathrm{CW}$ mode. We will give a more detailed description in the following two sessions.

\section{A. Epitaxial structire}

The epitaxial structures of quantum dot lasers were deposited by Veeco solid-source MBE GEN 930. After the preparation of offcut Si substrate, a thin film of AlAs nucleation layer was deposited on Si substrate by migration enhanced epitaxy (MEE) technique at relative low temperature about $350^{\circ} \mathrm{C}$. As AlAs nucleation layer suppressed the threedimensional growth, a good interface was formed for the following growth. After this, GaAs buffer was formed by threestep growth technique in different growth temperature. First, 30 $\mathrm{nm} \mathrm{GaAs}$ was deposited at the same temperature as AlAs; then, $170 \mathrm{~nm} \mathrm{GaAs}$ film was formed afterwards at higher temperature $450^{\circ} \mathrm{C}$; at the end, $800 \mathrm{~nm}$ high temperature $\mathrm{GaAs}$ was deposited at $590^{\circ} \mathrm{C}$. After depositing the GaAs buffer, $10 \mathrm{~nm}$ $\mathrm{In}_{0.18} \mathrm{Ga}_{0.82} \mathrm{As} / 10 \mathrm{~nm}$ GaAs SLS was used as DFLs to further reduce the threading dislocation density (TDD). Four repeats of DFLs were performed on GaAs buffer and each DFL included five periods of SLS and separated by $300 \mathrm{~nm}$ GaAs. In-situ thermal annealing was performed in each DFL after the formation of five periods of SLS. This will help to increase the mobility of TDs and then lead to the annihilation before the subsequently growth. Fig. 1 (a) (b) give a clear explanation on the TDD according to the different position of epitaxial structure. Most of the TDs were stopped at first $200 \mathrm{~nm}$ of the buffer layer. However, TDD is still at the level of $10^{9} \mathrm{~cm}^{-2}$ at position 1. After four repeats of DFLs, the TDD was clearly reduced and down to the level of $10^{5} \mathrm{~cm}^{-2}$ after the last $300 \mathrm{~nm}$ GaAs space layer. We successfully demonstrated a high-quality buffer for the following laser structure growth. Subsequently, a typical InAs/GaAs DWELL laser structure will be deposited on well optimized low TDD GaAs virtual substrate on Si.

\section{B. Device performance}

To date, we achieved low threshold current density and high optical output power quantum dot lasers on offcut Si substrate. 
Fig. 2 (insert) gives an AFM image of typical uncapped quantum dots layer grown under same condition with the laser devices. Good uniformity with $3 \times 10^{10} \mathrm{~cm}^{-2}$ quantum dot density is obtained. Fig. 2 also provides a photoluminescence (PL) image measured of the uncapped quantum dot layer which emitting at $\sim 1300 \mathrm{~nm}$ with full-width at half maximum (FWHM) $29 \mathrm{meV}$. Broad area lasers were fabricated based on the laser structure we developed. Eventually, we demonstrated a high reliability, high performance electrically pumped continuous wave $1.3 \mu \mathrm{m}$ QD laser on $4^{\circ}$ offcut $\mathrm{Si}(001)$ substrate with $\mathrm{J}_{\text {th }}$ of $62.5 \mathrm{~A} / \mathrm{cm}^{2}$ at room temperature and maximum operating temperature $75^{\circ} \mathrm{C}$ at $\mathrm{CW}$ mode. Fig. 3 shows the light-current-voltage (LIV) characteristic measurement for our InAs/GaAs quantum dot laser monolithically grown on Si. High optical output power of $105 \mathrm{~mW}$ was observed under injection current density of 650 $\mathrm{A} / \mathrm{cm}^{2}$.

\section{QUANTUM DOTS LASER MONOLITHICALLY INTEGRATED ON EXACT (001) SILICON SUBSTRATE}

Based on our previous discussion, we have successfully demonstrated the III-V quantum dot lasers grown on offcut $\mathrm{Si}$ substrate to prevent the formation of APBs. However, although the offcut substrate can annihilate the APBs caused by polar III$\mathrm{V}$ materials grown on non-polar group IV materials, it is not fully compatible to the industrial standard fabrication. Therefore, most recently, we have demonstrated the $1.3 \mu \mathrm{m}$ electrically pumped CW quantum dot lasers on exact Si (001) substrate which is industrial compatible. A $\mathbf{J}_{\text {th }}$ down to 425 $\mathrm{A} / \mathrm{cm}^{2}$ at room temperature with maximum operation temperature $36^{\circ} \mathrm{C}$ under $\mathrm{CW}$ mode was achieved [3]. Detailed Epitaxial structure and devices performance will be discussed in following sessions.

\section{A. Epitaxial structure}

The schematic diagram of our epitaxial structure on exact (001) Si substrate is shown in Fig. 4. $400 \mathrm{~nm} \mathrm{GaAs} \mathrm{buffer} \mathrm{was}$ grown in steps by MOCVD on an $300 \mathrm{~mm}$ diameter industrial standard Si (001) substrate without offcut. Firstly, a $40 \mathrm{~nm}$ thin film GaAs nucleation layer was deposited at low growth temperature $\left(400-500^{\circ} \mathrm{C}\right)$. Then, a $360 \mathrm{~nm}$ GaAs buffer was grown at higher temperature $\left(600-700^{\circ} \mathrm{C}\right)$ [4]. Eventually, as the AFM image shown in Fig. 5 (a), an APB free GaAs surface and small root mean square (RMS) surface roughness of $\sim 0.86$ $\mathrm{nm}$ were achieved. Afterwards, sample was moved to MBE chamber for the following laser structure growth. $600 \mathrm{~nm} \mathrm{GaAs}$ buffer was deposited firstly and following by five repeats of DFLs consist of five periods of optimized InGaAs/GaAs SLS separated by $300 \mathrm{~nm}$ GaAs. Then, a InAs/GaAs quantum dot laser structure was deposited on it started with $1.4 \mu \mathrm{m}$ n-type AlGaAs cladding layer and ended with $300 \mathrm{~nm}$ p-type GaAs contact layer. The active region consists of five repeats of undoped InAs/GaAs DWELL structure separated by $50 \mathrm{~nm}$ GaAs space layers.

\section{B. Device performance}

These quantum dot laser samples grown on exact (001) $\mathrm{Si}$ substrate were fabricated into broad area lasers and took various relative measurements. Fig. 5 (b) shows the room temperature PL comparison for these two samples. They present comparable emission intensity at $\sim 1285 \mathrm{~nm}$ with $\sim 32$ meV FWHM. The intensity of GaAs based quantum dots sample is about 1.3 times greater than $\mathrm{Si}$ (001) based sample. The surface morphology investigation was done by AFM for an uncapped InAs quantum dots layer grown on same condition with laser structure Fig. 6 presents the $1 \times 1 \mu^{2}$ AFM image of uncapped InAs QDs grown on Si (001) substrate which shows good uniformity and the dot density are $\sim 3.5 \times 10^{10} \mathrm{~cm}^{-2}$. The LIV characteristic comparison is presented in Fig. 7 , the $J_{\text {th }}$ is $210 \mathrm{~A} / \mathrm{cm}^{2}$ and $425 \mathrm{~A} / \mathrm{cm}^{2}$ for $\mathrm{GaAs}$ based, and $\mathrm{Si}$ based lasers respectively. The slope efficiencies are calculated as 0.12 W/A and $12.7 \%$ for GaAs based devices and $0.068 \mathrm{~W} / \mathrm{A}$ and $7.2 \%$ for the $\mathrm{Si}$ based devices. From Fig. 8, the maximum output power of single facet is $43 \mathrm{~mW}$ achieved under $1332 \mathrm{~A} / \mathrm{cm}^{2}$ injection current density at $\mathrm{CW}$ mode and only small rollover presented. For pulsed mode, the maximum output power is $134 \mathrm{~mW}$ under $2 \mathrm{kA} / \mathrm{cm}^{2}$ injection current density. Fig. 9 presents the emission spectrum under different injection current density under CW mode. When the injection current density increases to $425 \mathrm{~A} / \mathrm{cm}^{2}$, typical lasing behavior presented. Fig. 10-11 show the light-current (LI) characteristic under pulsed and $\mathrm{CW}$ mode respectively. Under pulsed mode, the characteristic temperature $\mathrm{T}_{0}$ is $\sim 32 \mathrm{~K}$ between $16^{\circ} \mathrm{C}$ to $102^{\circ} \mathrm{C}$. And for $\mathrm{CW}$ mode, lasing operation was observed when heatsink heated up to $36^{\circ} \mathrm{C}$.

\section{CONCLUSION}

We reviewed the recent results about III-V quantum dots lasers monolithically integrated on offcut $\mathrm{Si}$ and exact (001) $\mathrm{Si}$ substrate in this paper. They all present promising potential for high efficient and reliable on-chip light source for Si based optoelectronics integrated circuits.

\section{ACKNOWLEDGMENT}

This work was supported by UK Engineering and Physical Sciences Research Council (EPSRC) [grant numbers EP/J012904/1, EP/P000886/1, EP/P006973/1] and EPSRC National Epitaxy Facility; S.C. thanks for the Royal Academy of Engineering for funding his Research Fellowship.

\section{REFERENCES}

[1] T. Wang, H. Liu, A. Lee, F. Pozzi, and A. Seeds, "1.3- $\mu \mathrm{m}$ InAs/GaAs quantum-dot lasers monolithically grown on $\mathrm{Si}$ substrates," Opt. Express, vol. 19, pp. 11381-11386, 2011.

[2] S. Chen, W. Li, J. Wu, Q. Jiang, M. Tang, S. Shutts, S. Elliott, A. Sobiesierski, A. Seeds, I. Ross, P. Smowton, and H. Liu, "Electrically pumped continuous-wave III-V quantum dot lasers on silicon," Nat. Photonics, vol. 10, pp. 307-311, 2016.

[3] S. Chen, M. Liao, M. Tang, J. Wu, M. Martin, T. Baron, A. Seeds, and H. Liu, "Electrically pumped continuous-wave $1.3 \mu \mathrm{m}$ InAs/GaAs quantum dot lasers monolitically grown on on-axis Si (001) substrates," Opt. Express, vol. 5, pp. 4632-4639, 2017..

[4] R. Alcotte, M. Martin, J. Moeyaert, R. Cipro, S. David, F. Bassani, F. Ducroquet, Y. Bogumilowicz, E. Sanchez, Z. Ye, X. Bao, J. Pin, and T. Baron, "Epitaxial growth of antiphase boundary free GaAs layer on 300 mm Si (001) substrate by metalorganic chemical vapour deposition with high mobility," APL Mater. 4(4), 046101 (2016). 
(a)

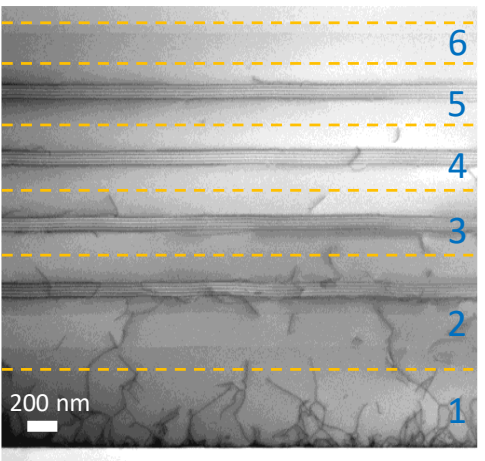

(b)

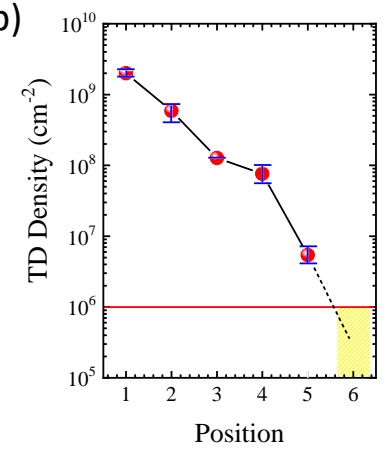

Fig. 1. (a) Bright-field scanning TEM image of DFLs; (b) Dislocation density measured at different positions in (a).

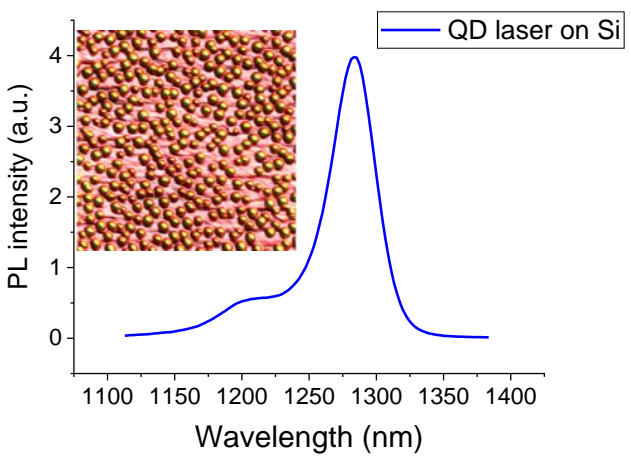

Fig. 2. PL spectrum for QD grown on Si emitting at $\sim 1300 \mathrm{~nm}$; Inset: representative AFM image of uncapped QD grown on $\mathrm{Si}$

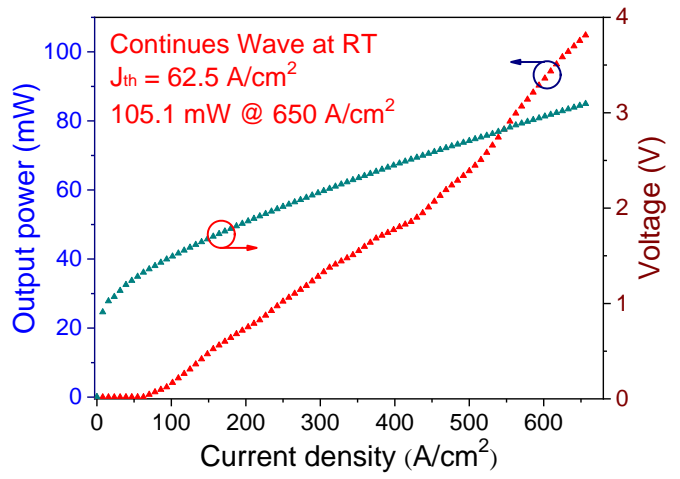

Fig. 3. LIV characteristics for a $50 \mu \mathrm{m} \times 3200 \mu \mathrm{m}$ InAs/GaAs $\mathrm{QD}$ laser grown on Si substrate under $\mathrm{CW}$ operation at $18^{\circ} \mathrm{C}$.

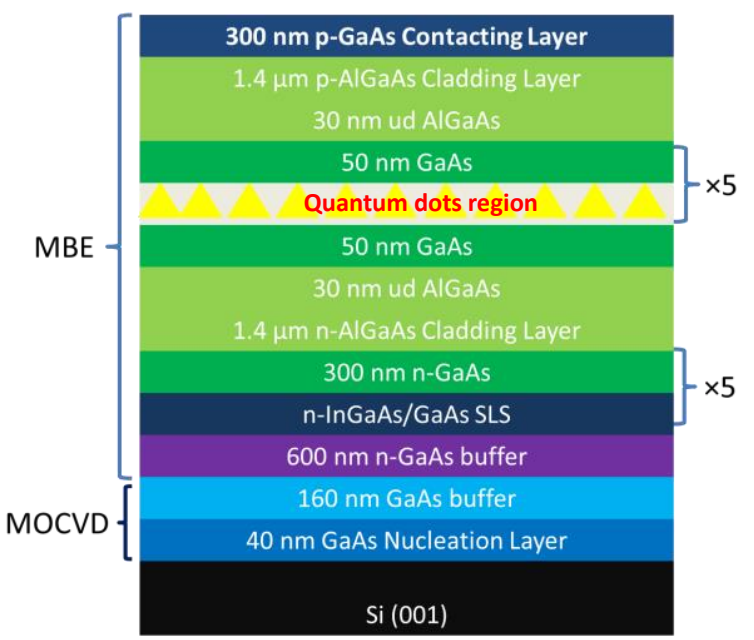

Fig. 4. The schematic diagram of the QD laser structure grown on the on-axis $\mathrm{Si}(001)$ substrate.
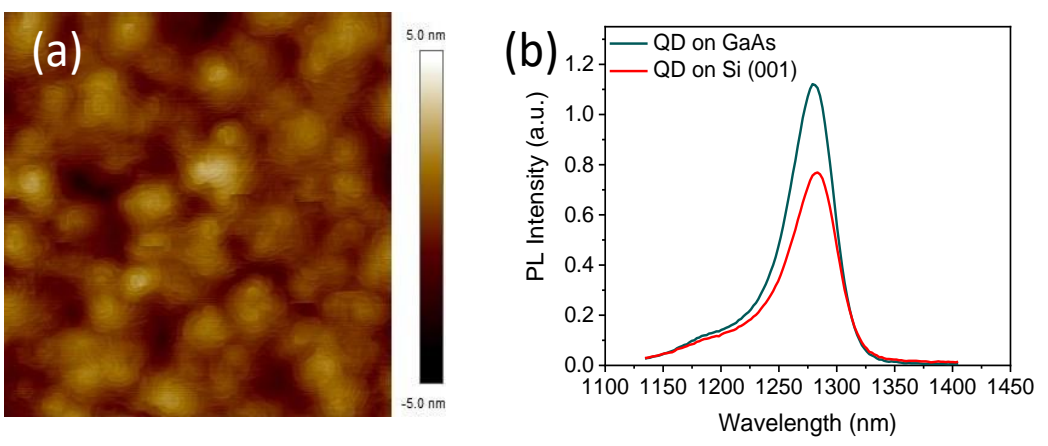

Fig. 5. (a) $5 \times 5 \mu \mathrm{m}^{2}$ AFM image of a $400 \mathrm{~nm}$ GaAs buffer direct grown on exact Si (001) substrate; (b) Room temperature PL comparison of InAs/GaAs QD laser structure grown on exact $\mathrm{Si}(001)$ and GaAs substrate.

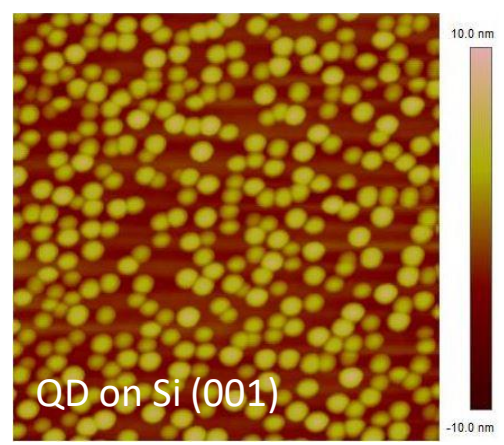

Fig. 6. $1 \times 1 \mu \mathrm{m}^{2}$ AFM image of uncapped InAs QDs grown on exact $\mathrm{Si}(001)$ substrate. 


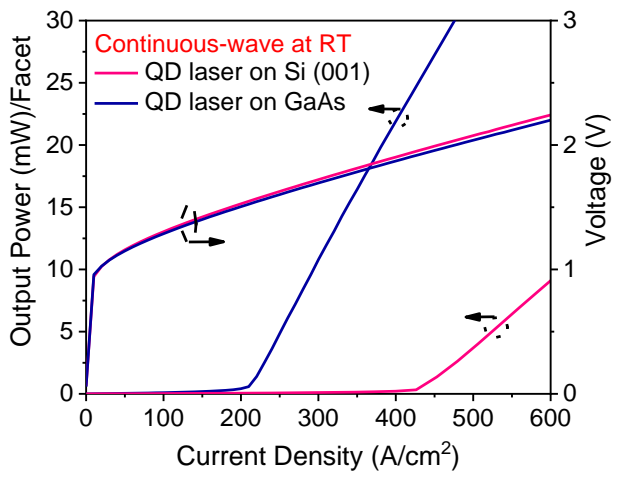

Fig. 7. LIV characteristic comparison of a InAs/GaAs QD laser grown on $\mathrm{Si}$ (001) and native GaAs substrate at room temperature under CW operation.

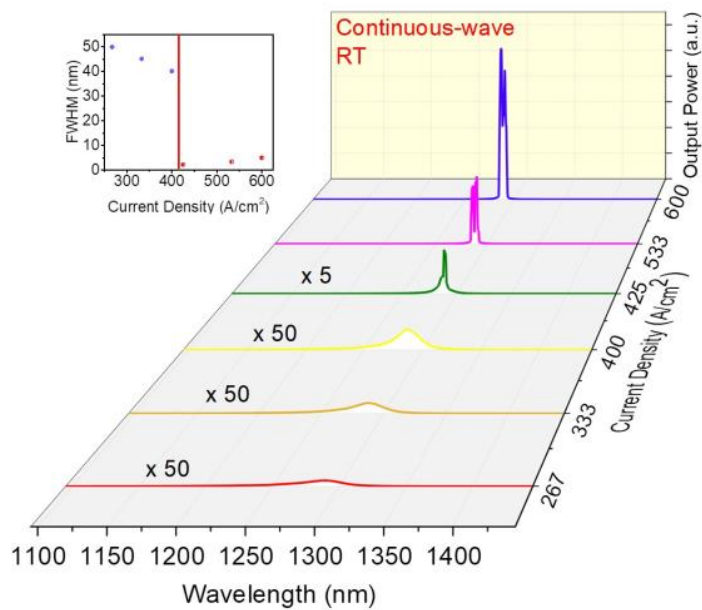

Fig. 9. Emission spectra for InAs/GaAs QD laser on Si (001) substrate at various injection current densities at room temperature. The inset shows the FWHM change as a function of injection current density.

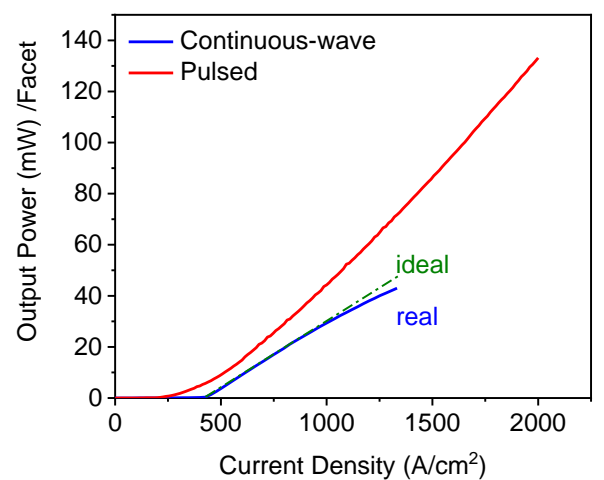

Fig. 8. LI comparison of InAs/GaAs QD laser grown on $\mathrm{Si}$ (001) substrate under $\mathrm{CW}$ and pulsed conditions at room temperature.

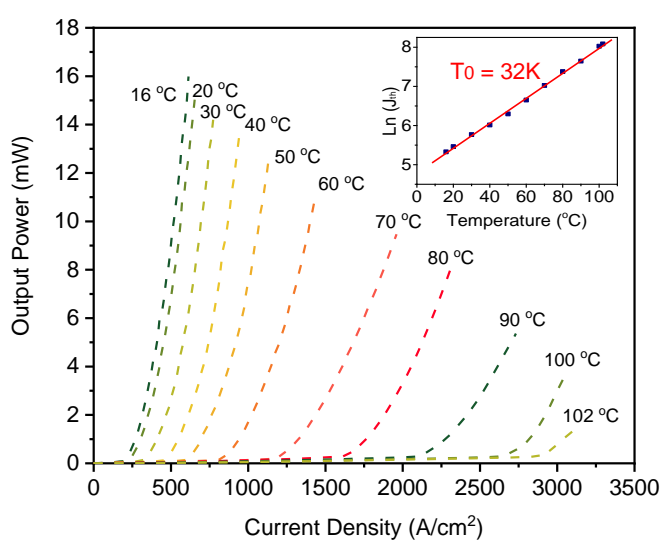

Fig. 10. Single facet LI curve for a InAs/GaAs QD laser grown on $\mathrm{Si}(001)$ substrate at different heat sink temperatures under pulsed condition. The inset shows the natural logarithm of current density against temperature in the ranges of $16-102^{\circ} \mathrm{C}$.

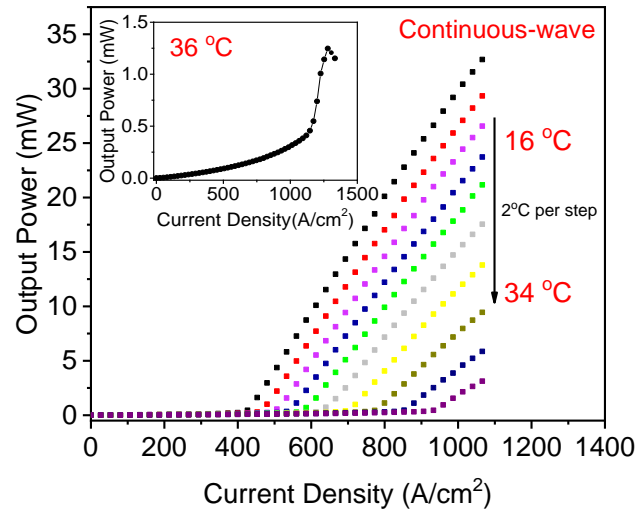

Fig. 11. Single facet LI curve for the same InAs/GaAs QD laser on $\mathrm{Si}(001)$ as a function of temperature under $\mathrm{CW}$ operation. The inset shows the LI curve for this QD laser at a heat sink temperature of $36^{\circ} \mathrm{C}$. 